\title{
Análisis de la gestión de protección y conservación del medio ambiente en los departamentos de la región de la selva, Perú
}

\author{
Analysis of environmental protection and conservation management in the departments of the jungle region, \\ Peru
}

\author{
Percy Ariasi ${ }^{1}$, Manuel Amarísta ${ }^{1}$
}

\section{RESUMEN}

El objetivo del estudio fue analizar la gestión de protección y conservación del medio ambiente desarrollado por la unidad ambiental (UA) de las municipalidades distritales de los departamentos de la región de la selva del Perú. Se desarrolló un estudio descriptivo, de corte transversal, y se usó los indicadores ambientales reportados por la Encuesta Nacional de Municipalidades 2019 del Instituto Nacional de Estadística e Informática. Los datos se organizaron en Microsoft Excel y analizados mediante estadística descriptiva con software Stata versión 15. Se determinó la existencia de una brecha entre el número de UA instaladas y las requeridas por departamentos, identificando mayor problema en los departamentos de Amazonas y Loreto. Las denuncias por tipo de contaminación más frecuentes fueron: sonora, de agua y aire. Los principales tipos de elementos contaminantes reportados fueron: crianza de animales domésticos sin control, aguas residuales, y deforestación y quema de bosques. El análisis de la coherencia entre atención de denuncias por tipo de contaminación, aplicación de instrumento de gestión y acciones de conservación mostró la existencia de UA que trabajaron de acuerdo a lo establecido en el marco legal (Ley $\mathrm{N}^{\mathrm{o}} 28611$ ), y otro grupo de UA que reciben denuncias, pero no aplicaron instrumentos de gestión ambiental y no realizan acciones de conservación, calificando este tipo de gestión ambiental como deficiente. La definición de una estrategia de intervención ante esta situación, pasa por fiscalizar la aplicación de normas y leyes vigentes, impulsar la participación ciudadana, y trabajar sobre: tipos y elementos de contaminación más frecuentes.

PALABRAS CLAVE: ambiente, conservación, gestión ambiental, salud ambiental, selva

\section{SUMMARY}

The objective of the study was to analyze the environmental protection and conservation management developed by the environmental unit (EU) of the district municipalities of the departments of the jungle region of Peru. A descriptive, cross-sectional study was developed, and the environmental indicators reported by the National Survey of Municipalities 2019, of the National Institute of Statistics and Informatics were used. The data were organized in Microsoft Excel and analyzed using descriptive statistics with Stata version 15. The existence of a gap between the number of EU installed and those required by departments was determined, identifying a greater problem in Amazonas and Loreto. The most frequent complaints by type of contamination were: noise, water and air pollution. The main types of pollutants reported were: uncontrolled domestic animal husbandry, sewage, and deforestation and forest burning. The analysis of the coherence between the attention of complaints by type of contamination, application of management instruments and conservation actions showed the existence of EUs that worked in

Facultad de Medicina Veterinaria y Zootecnia, Universidad Peruana Cayetano Heredia 
accordance with the legal framework (Law No. 28611), and another group of EUs that received complaints but did not apply environmental management instruments and did not carry out conservation actions. This type of environmental management was rated as deficient. The definition of an intervention strategy to deal with this situation involves monitoring the application of current laws and regulations, promoting citizen participation, and working on the most frequent types and elements of contamination.

KEY WORDS: Environment, management, conservation, protection, environmental health and Peru.

\section{INTRODUCCIÓN}

La contaminación ambiental es un proceso continuo y cíclico de alteraciones nocivas que involucra todos los ambientes: aire, agua y suelo. Esta puede ser ocasionada por causas naturales como la presencia de material inorgánico (ejemplo arsénico en fuentes de aguas (ríos y pozos) o las generadas por desastres naturales (terremotos, tsunamis, erupción de volcanes, etc.) (Albert, 2004; Gonzales et al., 2014); o por causas artificiales, vinculadas con el desequilibrio de la relación entre el modelo de producción económico (industrializado) y la conservación de los ecosistemas. En este grupo se encuentran los agentes biológicos (descomposición y fermentación), físicos (ruido, calor, radioactividad, etc.) y químicos (productos generados en las industrias, como: derivados del petróleo, pesticidas, plásticos, ácidos, etc.) (Albert, 2004).

Los problemas ambientales se fueron agravando en el tiempo como consecuencia de la enorme irracionalidad e ineficacia de la mayor parte de las estrategias productivas y tecnológicas, que impactaron negativamente sobre los componentes, procesos y ritmos naturales de los ecosistemas (Vargas y Velázquez, 2014). Esta situación impulsó el desarrollo de un gran debate internacional que conllevó con la realización de la "Conferencia de Naciones Unidas sobre Ambiente Humano y Desarrollo", la cual se llevó a cabo en el año 1972 en la ciudad de Estocolmo - Suecia. La finalidad de este evento fue darle a la problemática ambiental una dimensión universal y proponer soluciones comunes a problemas diferentes (Guzmán, 2012). De esta forma se dio origen a la política ambiental internacional cuyo fin ha sido establecer una relación saludable entre hombre sociedad y los modelos de producción (Vargas, 2008).

En este sentido, en la actualidad la respuesta a la problemática ambiental tiene como eje central de desarrollo, los recursos sociales y naturales, sin dejar de lado, el desarrollo económico sostenible y sustentable, en cada región o país (Fernández, 2020). Ante esta situación, los países han ido adecuando sus políticas y legislaciones ambientales a estos nuevos desafíos, y se han comprometido con la ejecución de estrategias de mitigación a largo plazo (Ministerio de la Producción, 2014).

El Perú ha realizado una serie de reformas en busca de alinearse con los compromisos internacionales. Así, la Constitución Política del Perú vigente, en sus artículos $66^{\circ}, 67^{\circ}, 68^{\circ}$ y $69^{\circ}$, dispone que el Estado promueve el uso sostenible de sus recursos naturales. Además, está obligado a promover la conservación de la diversidad biológica de las áreas naturales protegidas, así como el desarrollo sostenible de la Amazonía, con una legislación adecuada; la Ley $\mathrm{N}^{\circ}$ 28245 del Sistema Nacional de Gestión Ambiental y la Ley $\mathrm{N}^{\circ} 29050$ (modificatoria de la Ley $\mathrm{N}^{\circ} 28245$, designa a los gobiernos locales como los responsables de la formulación e implementación del sistema de gestión ambiental; y desde el 2015, la Organización de las Naciones Unidas (ONU) aprobó la Agenda 2030 sobre el Desarrollo Sostenible, y Perú se ha comprometido con su ejecución, seguimiento $\mathrm{y}$ evaluación de los progresos (Ministerio del Ambiente [MINAM], 2016a). En coherencia con esta reforma, desde el $1^{\circ}$ de enero de 2016 el Perú ha establecido su línea base, y ha empezado a orientar los Objetivos de Desarrollo Sostenible (ODS) a sus políticas públicas (MINAM, 2016b).

Con respecto a la gestión de protección y conservación del ambiente en Perú, a nivel local el ente rector es la oficina o unidad ambiental (UA). Según el Instituto Nacional de Estadística e Informática [INEI] (2019) esta: "tienen la responsabilidad de mejorar la protección, fiscalización y manejo de la información ambiental; así como el uso de los recursos naturales, entre otros temas que contribuyen a optimizar las condiciones de vida de las personas". Asimismo, el país viene impulsando el desarrollo de la línea política "la gestión ambiental integral". Por tanto, en la actualidad se les exige a las municipalidades distritales que se avoquen al avance e implementación de las UA y la legislación vigente (INEI, 2017). 
En relación a los departamentos de la región de la selva del Perú, no todas las municipalidades distritales poseen UA, lo que representa una brecha entre sus departamentos, y a su vez imposibilita el cumplimiento de la legislación vigente. Por tanto, el objetivo del estudio fue analizar la gestión de protección y conservación del medio ambiente desarrollado por las UA de las municipalidades distritales de los departamentos de la región de la selva del Perú, considerando los indicadores ambientales reportados por la Encuesta Nacional de Municipalidades 2019.

\section{MATERIAL Y MÉTODOS}

La investigación correspondió a un estudio observacional, descriptivo de corte transversal que utilizó información de fuentes secundarias (base de datos) pertenecientes a la Encuesta Nacional de Municipalidades 2019. Este instrumento fue aplicado por el INEI. Así, la población objetivo estuvo representada por las respuestas de los encuestados procedentes de todas las municipalidades distritales de los cinco departamentos de la región de la selva del Perú (Amazonas $=84$ distritos; Loreto $=53$ distritos; Madre de Dios = 11 distritos; San Martin = 77 distritos; y Ucayali $=17$ distritos).

La variable dependiente fue la gestión de protección $\mathrm{y}$ conservación del ambiente, y como variables independientes se consideraron las siguientes:

a. Presencia de oficina o unidad ambiental en la municipalidad (si o no);

b. Número de denuncias ambientales recepcionadas por las municipalidades;

c. Número de denuncias ambientales atendidas por las municipalidades;

d. Tipos de los elementos que originan la contaminación ambiental (Presencia o ausencia).

- Emanación de gases y partículas de fábricas y refinerías;

- Emisión de gases de vehículos motorizados;

- Relaves mineros;

- Ruidos de alta intensidad;

- Aguas residuales;

- Acumulación y quema clandestina de basura y rastrojos;

- Deforestación y quema de bosques; y

- Crianza de animales domésticos sin control.

e. Instrumentos de gestión ambiental que poseen las municipalidades (poseen o No Poseen),

- Política ambiental local;

- Diagnóstico ambiental local;

- $\quad$ Plan de acción ambiental local;
- Agenda ambiental local;

- Plan anual de evaluación y fiscalización ambiental;

- Plan nacional de educación ambiental;

- Plan de acción en género y cambio climatológico;

- Estrategia nacional de bosques y cambio climático; y

- Plan director de áreas naturales protegidas.

f. Acciones para incentivar la gestión ambiental (realizan o no realizan),

- Campañas de concientización ambiental;

- Concursos de iniciativas de cuidado del ambiente;

- $\quad$ Ejecución de proyectos ambientales;

- Comisión ambiental municipal;

- Apoyo en formulación de proyectos sobre problemática ambiental;

- Convenios interinstitucionales;

- Charlas educativas;

- Atención y apoyo a denuncias ambientales por la población; y

- Mediciones acústicas con sonómetro.

Estos datos se encuentran disponible al público en la página Web del INEI, de donde se realizó su descarga. Asimismo, los módulos y la documentación de la encuesta del INEI se encontró en formatos compatibles y de amplia divulgación en el mercado (SPSS, Microsoft Excel, Acrobat Reader). Por tanto, los datos se lograron recuperar en el formato Excel, y el diccionario de variables estudiadas en Acrobat Reader.

Para el procesamiento y análisis descriptivo de los datos se usó el software Stata (versión 15). Así, los resultados se presentaron en cuadros para describir: el número municipalidad distrital que cuentas con UA por provincias y departamento; los tipos de denuncias y elementos por contaminación más frecuentes por departamento; y el número de denuncias recibidas y atendidas en las UA que tienen o no instrumentos de gestión y realizaron o no acciones de conservación ambiental.

\section{RESULTADOS}

La revisión de la base de datos de la Encuesta Nacional de Municipalidades 2019, permitió determinar una gran brecha entre el número existente de UA (149) y el total de municipalidades distritales (242), contabilizando el total de provincias (24) y departamentos (5) de la región de la selva. Este déficit 
o faltante de UA por distritos y provincias, es mayor en los departamentos de Amazonas y Loreto, pero es menor en Madre de Dios, San Martín y Ucayali, donde se observa que el promedio de presencias de las UA por distrito corresponde al esperado, es decir, cercano a 1. Además, se observa que no hay una buena distribución geográfica, ya que algunos departamentos concentran mayor número de UA por distritos y provincias. Los detalles de estos resultados se presentan en la tabla 1 .

Por otro lado, se logró determinar la frecuencia absoluta de denuncias recibidas y atendidas por tipo de contaminación en las UA de las municipalidades distritales, predominando la sonora (201), aire (193), agua (161), suelo (113), y deforestación y quema de bosques (47) (tabla 2). Asimismo, los tipos de elementos contaminantes reportados como más frecuencia fueron crianza de animales domésticos sin control (128), aguas residuales (125), deforestación $\mathrm{y}$ quema de bosques (121) y acumulación y quema clandestina de rastrojos (121) (tabla 3). En consecuencia, los departamentos que se reportan más afectados por estos elementos fueron San Martín, Amazonas y Loreto (tabla 3).

En relación a los instrumentos de gestión ambiental, se determinó que de las 149 UA existentes en la región de la selva para el 2019, sólo el 87,91\% (131) de estas informaron tener instrumentos de gestión. Asimismo, en el Cuadro 4 se describe el número de UA por departamentos y los tipos instrumentos que se usaron durante el periodo de estudio.

También, al cuantificar la frecuencia absoluta de las acciones realizada por tipo, para incentivar la conservación del ambiente por las UA de los departamentos seleccionadas, se logró identificar dos grupos: 1. Acciones más frecuentes: a. Disponen de Comisión Ambiental Municipal (172); b. Charlas Educativas (129); y c. Campaña de concientización ambiental (102); 2. Menos frecuente: a. Atención y apoyo a denuncias ambientales por la población

Tabla 1. Municipalidades distritales por departamentos de la región de la selva que cuentan con oficina o unidad ambiental para recepcionar y atender denuncias por tipo de contaminación, reportadas en Encuesta Nacional de Municipalidades 2019.

\begin{tabular}{|c|c|c|c|c|c|}
\hline \multirow[b]{2}{*}{ Variables } & \multicolumn{5}{|c|}{ Departamentos de la región de la selva } \\
\hline & Amazonas & Loreto & $\begin{array}{l}\text { Madre de } \\
\text { Dios }\end{array}$ & $\begin{array}{c}\text { San } \\
\text { Martín }\end{array}$ & Ucayali \\
\hline $\mathrm{N}^{\mathrm{o}}$ de provincias & 7 & 8 & 3 & 10 & 4 \\
\hline $\mathrm{N}^{\mathrm{o}}$ de provincias con unidad ambiental & 6 & 7 & 1 & 8 & 2 \\
\hline $\mathrm{N}^{\mathrm{o}}$ de distritos & 84 & 53 & 11 & 77 & 17 \\
\hline $\mathrm{N}^{\mathrm{o}}$ de distritos con unidad ambiental & 30 & 28 & 10 & 64 & 17 \\
\hline $\begin{array}{l}\text { Promedio de unidad ambiental por } \\
\text { provincias }\end{array}$ & 5 & 4 & 10 & 8 & 8,5 \\
\hline $\begin{array}{l}\text { Promedio de unidad ambiental por } \\
\text { distritos }\end{array}$ & 0,35 & 0,52 & 0,9 & 0,83 & 1 \\
\hline
\end{tabular}

Tabla 2. Departamentos de la región de la selva con unidad ambiental, que recepcionó y atendió denuncias distribuido por tipos de contaminación. Encuesta Nacional de Municipalidades 2019.

\begin{tabular}{|c|c|c|c|c|c|c|c|c|c|c|c|}
\hline \multirow{2}{*}{$\begin{array}{l}\text { Tipos de } \\
\text { contaminación }\end{array}$} & \multirow{2}{*}{$\begin{array}{c}\begin{array}{c}\text { Total de } \\
\text { denuncias }\end{array} \\
\mathrm{N}^{\circ} \\
\end{array}$} & \multicolumn{2}{|c|}{ Amazonas } & \multicolumn{2}{|c|}{ Loreto } & \multicolumn{2}{|c|}{$\begin{array}{c}\text { Madre de } \\
\text { Dios }\end{array}$} & \multicolumn{2}{|c|}{ San Martín } & \multicolumn{2}{|c|}{ Ucayali } \\
\hline & & $\mathrm{n}$ & $\%$ & $\mathbf{n}$ & $\%$ & $\mathrm{n}$ & $\%$ & $\mathbf{n}$ & $\%$ & n & $\%$ \\
\hline Sonora & 201 & 22 & 10,9 & 41 & 20,4 & 66 & 32,8 & 35 & 17,4 & 37 & 18,4 \\
\hline Aire & 193 & 8 & 4,1 & 28 & 14,5 & 32 & 16,6 & 47 & 24,4 & 78 & 40,4 \\
\hline Agua & 161 & 24 & 14,9 & 56 & 34,8 & 10 & 6,2 & 55 & 34,2 & 16 & 9,9 \\
\hline Suelo & 113 & 22 & 19,5 & 20 & 17,7 & 36 & 31,9 & 31 & 27,4 & 4 & 3,5 \\
\hline $\begin{array}{l}\text { Deforestación y quema } \\
\text { de bosques }\end{array}$ & 47 & 6 & 12,8 & 14 & 29,8 & 5 & 10,6 & 22 & 46,8 & 0 & 0 \\
\hline Total & 715 & 82 & 11,5 & 159 & 22.2 & 149 & 20,8 & 190 & 26,6 & 135 & 18,9 \\
\hline
\end{tabular}


Tabla 3. Departamentos de la región de la selva con unidad ambiental, que recepcionó y atendió denuncias por tipos de elementos contaminantes. Encuesta Nacional de Municipalidades 2019.

\begin{tabular}{|c|c|c|c|c|c|c|c|c|c|c|c|}
\hline \multirow{2}{*}{$\begin{array}{l}\text { Tipo de elemento } \\
\text { contaminante }\end{array}$} & \multirow{2}{*}{$\begin{array}{c}\text { Total de } \\
\text { denuncias }\end{array}$} & \multicolumn{2}{|c|}{ Amazonas } & \multicolumn{2}{|c|}{ Loreto } & \multicolumn{2}{|c|}{$\begin{array}{l}\text { Madre de } \\
\text { Dios }\end{array}$} & \multicolumn{2}{|c|}{ San Martín } & \multicolumn{2}{|c|}{ Ucayali } \\
\hline & & $\mathbf{n}$ & $\%$ & $\mathbf{n}$ & $\%$ & $\mathbf{n}$ & $\%$ & n & $\%$ & $\mathbf{n}$ & $\%$ \\
\hline $\begin{array}{l}\text { Emanación de gases y } \\
\text { partículas de fábricas o } \\
\text { refinerías }\end{array}$ & 16 & 1 & 6,3 & 4 & 25 & 1 & 6,3 & 6 & 37,5 & 4 & 25 \\
\hline $\begin{array}{l}\text { Emisión de gases de } \\
\text { vehículos motorizados }\end{array}$ & 59 & 12 & 20,3 & 16 & 27,1 & 1 & 1,7 & 22 & 37,3 & 8 & 13,6 \\
\hline Relaves mineros & 6 & 1 & 16,7 & 2 & 33,3 & 2 & 33,3 & 0 & 0 & 1 & 16,7 \\
\hline $\begin{array}{l}\text { Ruidos con alta intensidad } \\
\text { que } \\
\text { dañan la salud }\end{array}$ & 47 & 9 & 19,1 & 14 & 29,8 & 2 & 4,3 & 18 & 38,3 & 4 & 8,5 \\
\hline Aguas residuales & 125 & 31 & 24,8 & 27 & 21,6 & 5 & 4 & 50 & 40 & 12 & 9,6 \\
\hline $\begin{array}{l}\text { Acumulación y quema } \\
\text { clandestina de basura y } \\
\text { rastrojos }\end{array}$ & 102 & 29 & 28,4 & 25 & 24,5 & 5 & 4,9 & 34 & 33,3 & 9 & 8,8 \\
\hline $\begin{array}{l}\text { Deforestación y quema de } \\
\text { bosques }\end{array}$ & 121 & 31 & 25,6 & 22 & 18,2 & 4 & 3,3 & 53 & 43,8 & 11 & 9,1 \\
\hline $\begin{array}{l}\text { Crianza de animales } \\
\text { domésticos sin control }\end{array}$ & 128 & 46 & 35,9 & 19 & 14,8 & 4 & 3,1 & 47 & 36,7 & 12 & 9,4 \\
\hline Total & 604 & 160 & 26,5 & 129 & 21,4 & 24 & 4 & 230 & 38,1 & 61 & 10,1 \\
\hline
\end{tabular}

Tabla 4. Departamentos de la región de la selva con unidades ambientales que señalaron tener instrumentos de gestión ambiental. Encuesta Nacional de Municipalidades 2019.

\begin{tabular}{|c|c|c|c|c|c|c|c|c|c|c|}
\hline \multirow[t]{2}{*}{$\begin{array}{l}\text { Instrumentos de } \\
\text { gestión ambiental }\end{array}$} & \multicolumn{2}{|c|}{$\begin{array}{l}\text { Amazonas } \\
(\mathbf{U A}=\mathbf{2 7})\end{array}$} & \multicolumn{2}{|c|}{$\begin{array}{c}\text { Loreto } \\
(\mathrm{UA}=\mathbf{2 5})\end{array}$} & \multicolumn{2}{|c|}{$\begin{array}{c}\text { Madre } \\
\text { de Dios } \\
(\mathrm{UA}=10)\end{array}$} & \multicolumn{2}{|c|}{$\begin{array}{l}\text { San Martín } \\
(\mathbf{U A}=\mathbf{5 5})\end{array}$} & \multicolumn{2}{|c|}{$\begin{array}{c}\text { Ucayali } \\
(\mathrm{UA}=14)\end{array}$} \\
\hline & $\mathbf{n}$ & $\%$ & n. & $\%$ & n. & $\%$ & $\mathrm{n}$ & $\%$ & $\mathbf{n}$ & $\%$ \\
\hline $\begin{array}{l}\text { Política ambiental } \\
\text { local }\end{array}$ & 6 & 22,2 & 4 & 16,0 & 4 & 40,0 & 24 & 43,6 & 5 & 35,7 \\
\hline $\begin{array}{l}\text { Diagnóstico } \\
\text { ambiental local }\end{array}$ & 6 & 22,2 & 4 & 16,0 & 5 & 50,0 & 20 & 36,4 & 4 & 28,6 \\
\hline $\begin{array}{l}\text { Plan de acción } \\
\text { ambiental local }\end{array}$ & 6 & 22,2 & 3 & 12,0 & 1 & 10,0 & 18 & 32,7 & 5 & 35,7 \\
\hline $\begin{array}{l}\text { Agenda ambiental } \\
\text { local }\end{array}$ & 5 & 18,5 & 5 & 20,0 & 5 & 50,0 & 11 & 20,0 & 4 & 28,6 \\
\hline $\begin{array}{l}\text { Plan anual de } \\
\text { evaluación y } \\
\text { fiscalización } \\
\text { ambiental }\end{array}$ & 25 & 92,6 & 24 & 96,0 & 8 & 80,0 & 46 & 83,6 & 14 & 100,0 \\
\hline $\begin{array}{l}\text { Plan nacional de } \\
\text { educación ambiental }\end{array}$ & 4 & 14,8 & 3 & 12,0 & 2 & 20,0 & 16 & 29,1 & 6 & 42,9 \\
\hline $\begin{array}{l}\text { Plan director de } \\
\text { áreas naturales } \\
\text { protegidas }\end{array}$ & 0 & 0,0 & 0 & 0,0 & 1 & 10,0 & 2 & 3,6 & 1 & 7,1 \\
\hline
\end{tabular}


Tabla 5. Departamentos de la región de la selva con unidades ambientales que indicaron realizar acciones de conservación ambiental. Encuesta Nacional de Municipalidades 2019.

\begin{tabular}{|c|c|c|c|c|c|c|c|c|c|c|}
\hline \multirow{2}{*}{$\begin{array}{c}\text { Acciones de } \\
\text { Conservación } \\
\text { Ambiental }\end{array}$} & \multicolumn{2}{|c|}{$\begin{array}{c}\text { Amazonas } \\
(\mathrm{UA}=\mathbf{2 3})\end{array}$} & \multicolumn{2}{|c|}{$\begin{array}{c}\text { Loreto } \\
(\mathrm{UA}=\mathbf{2 2})\end{array}$} & \multicolumn{2}{|c|}{$\begin{array}{l}\text { Madre de Dios } \\
\quad(\mathrm{UA}=10)\end{array}$} & \multicolumn{2}{|c|}{$\begin{array}{c}\text { San Martín } \\
(\mathrm{UA}=56)\end{array}$} & \multicolumn{2}{|c|}{$\begin{array}{c}\text { Ucayali } \\
(\mathrm{UA}=13)\end{array}$} \\
\hline & $\mathbf{S i}$ & $\%$ & $\mathbf{S i}$ & $\%$ & Si & $\%$ & Si & $\%$ & $\mathbf{S i}$ & $\%$ \\
\hline $\begin{array}{l}\text { Campañas de } \\
\text { concientización } \\
\text { ambiental }\end{array}$ & 15 & 65,2 & 15 & 68,2 & 4 & 40 & 43 & 76,8 & 10 & 76,9 \\
\hline $\begin{array}{l}\text { Concursos de iniciativas } \\
\text { de cuidado del ambiente }\end{array}$ & 4 & 17,4 & 4 & 18,2 & 3 & 30 & 14 & 25 & 5 & 38,5 \\
\hline $\begin{array}{l}\text { Ejecutó proyectos } \\
\text { ambientales }\end{array}$ & 4 & 17,4 & 4 & 18,2 & 3 & 30 & 10 & 17,9 & 1 & 7,7 \\
\hline $\begin{array}{l}\text { Disponen de Comisión } \\
\text { Ambiental Municipal }\end{array}$ & 11 & 47,8 & 3 & 13,6 & 4 & 40 & 19 & 33,9 & 2 & 15,4 \\
\hline $\begin{array}{l}\text { Apoyó en la formulación } \\
\text { de proyectos sobre }\end{array}$ & 4 & 17,4 & 4 & 18,2 & 0 & 0 & 6 & 10,7 & 3 & 23,1 \\
\hline Problemática ambiental & 6 & 26,1 & 19 & 86,4 & 5 & 50 & 13 & 23,2 & 3 & 23,1 \\
\hline Charlas educativas & 5 & 21,7 & 5 & 22,7 & 4 & 40 & 48 & 85,7 & 9 & 69,2 \\
\hline $\begin{array}{l}\text { Atención y apoyo a } \\
\text { denuncias ambientales } \\
\text { por la población }\end{array}$ & 11 & 47,8 & 12 & 54,5 & 2 & 20 & 25 & 44,6 & 5 & 38,5 \\
\hline $\begin{array}{l}\text { Realizó mediciones } \\
\text { acústicas con sonómetro }\end{array}$ & 4 & 17,4 & 5 & 22,7 & 2 & 20 & 7 & 12,5 & 2 & 15,4 \\
\hline
\end{tabular}

(65); b. Ejecución de proyectos ambientales (41); c. Problemática ambiental (33); y d. Concursos de iniciativas de cuidado del ambiente (32).

Por último, al analizar el nivel de coherencia entre las acciones de conservación ambiental realizadas, instrumentos de gestión y denuncias atendidas por tipo de contaminación en la gestión de las UA de cada municipalidad distrital de los departamentos seleccionados, encuentra dos situaciones que pueden ser identificadas a partir de las tablas 4 y 5 , es decir: 1. Oficinas con instrumentos de gestión y acciones de conservación realizadas, que atienden las denuncias recibidas en los departamentos; y 2. Oficinas que no tienen instrumentos de gestión y no realizan acciones de conservación, pero atienden denuncias por tipo de contaminación.

En relación a lo descrito en el párrafo anterior, se identificó que: 1. Los departamentos de Amazonas, Loreto, Madre de Dios, San Martín y Ucayali presentan un número de UA que no cuentan con los siguientes instrumentos: a. Política ambiental local; b. Diagnóstico ambiental local; c. Plan de acción ambiental local; d. Agenda ambiental local; e. Plan nacional de educación ambiental; y f. Plan director de áreas naturales protegidas. Asimismo, en estos departamentos algunas acciones de conservación ambiental no son llevadas a cabo por algunas UA, entre las que se identifican las siguientes: a. Concursos de iniciativas de cuidado del ambiente; b. Ejecutó proyectos ambientales; c. Disponen de comisión ambiental municipal; d. Apoyó en la formulación de proyectos sobre Problemática ambiental; y e. No realizó mediciones acústicas con sonómetro; y sólo 2. Amazonas, Loreto, Madre de Dios no realizan charlas educativas.

\section{DISCUSIÓN}

La gestión de protección y conservación del ambiente como una política del estado peruano es dirigida por su órgano rector, el Ministerio del Ambiente y se ejecuta en coordinación con los tres niveles de gestión administrativa del país, es decir, gobierno local, provincial, y departamental, en conformidad con la legislación ambiental vigente. Dentro de este contexto y tomando como referencia lo indicado en la Ley $\mathrm{N}^{\circ} 28245$ del Sistema Nacional de Gestión Ambiental y de la Ley Nº 29050 (modificatoria 
de la Ley $\mathrm{N}^{\circ}$ 28245), desde los años 2004 y 2007 respectivamente, se designan a los gobiernos locales como los responsables de la formulación e implementación del Sistema de Gestión Ambiental (MINAM, 2016b).

A pesar de contarse con un marco legal, como se indicado en el párrafo anterior, el análisis de los datos de la Encuesta Nacional del INEI 2019, coloca en evidencia que a nivel nacional no hubo un avance en cuanto a la instalación y funcionamiento de las unidades ambientales (UA), porque en el 2019 se instalaron 1 mil 146 de un total de 1 mil 874 municipalidades, representando un $61.2 \%$, es decir, que se aumentó en 43 oficinas con respecto al año 2018, donde se habían instalado 1 mil 180 de 1 mil 872 municipalidades que representaron un 65.3\% (INEI, 2019). Esta situación es similar, para algunos departamentos de la región de la selva, ya que no hubo un aumento sobre el porcentaje de instalación de UA. De esta forma, en esta región se identificó la siguiente situación: Amazonas (2018: $\mathrm{UA}=41.7 \%$; 2019: $\mathrm{UA}=35.7 \%$ ); y Loreto (2018: $\mathrm{UA}=64.2 \% ; 2019$ : $\mathrm{UA}=52.8 \%$ ). Sin embargo, el número de UA instaladas se mantiene en Madre de Dios (UA=90.9\%); y San Martín (UA=83.1\%), y se evidencia un aumenta en Ucayali (2018: UA $=76.5 \%$; 2019: $\mathrm{UA}=100 \%$ ).

Por otro lado, al contrastar las denuncias por tipo de contaminación atendidas a nivel nacional con respecto a la región de la selva, se observó la misma tendencia para estos años (2018 y 2019), sobre la frecuencia de denuncias recibidas y atendidas, es decir, que en el orden de las más frecuentes se encontraron: sonora, aire, agua, suelo y deforestación y quema de bosques (INEI, 2019). Pero, al revisar los tipos de contaminación que más afectación ocasionan a los departamentos seleccionados, se logra definir otro orden de importancia, ubicando en el primer lugar al tipo sonora, y en segundo al agua y aire. Asimismo, al contrastar las denuncias por tipos de elementos contaminantes entre estos dos niveles administrativos (nacional y región de la selva), se observa una coincidencia, ya que, son los mismos elementos contaminantes que se reportan tanto para el 2018 como en el 2019 (INEI, 2018, 2019). Por tanto, cualquier estrategia de intervención que se diseñe a nivel nacional o local para la protección y conservación del ambiente, debe considerar el trabajo sobre: 1. Crianza de animales domésticos sin control; 2. Aguas residuales; 3. Deforestación y quema de bosques; 4 . Acumulación y quema clandestina de rastrojos; y 5. Emisión de gases de vehículos motorizados.
$\mathrm{Al}$ revisar la coherencia entre la denuncia atendida por las oficinas de municipalidades distritales, los instrumentos de gestión con que cuentan, las acciones que realizan, estos muestran concordancia con el marco legal, es decir, a lo indicado en la Ley $\mathrm{N}^{\circ} 28611$ : "Ley General del Ambiente" (en el artículo 16: "De los instrumentos"; y articulo 17: "De los tipos de instrumentos"). Donde se señalas que estos son los mecanismos que orientan la ejecución de la "Política Nacional Ambiental". Además, el incentivo de las acciones de conservación y preservación del ambiente debe ser impulsado por los gobiernos locales, lo cual está en coherencia con lo que se define en el artículo 8 sobre la "Política Nacional Ambiental". No obstante, el análisis sobre los datos de la Encuesta del 2019 permitió identificar dos situaciones en las áreas de estudio: 1. Oficinas con instrumentos de gestión y acciones de conservación realizadas, que atienden las denuncias que se reciben en los departamentos; y 2. Oficinas que no tienen instrumentos de gestión y no realizan acciones de conservación, pero atienden denuncias por tipo de contaminación. En este último punto, se evidencia el nivel de incoherencia en el que se encuentran funcionando las UA a nivel de las municipalidades distritales por departamento.

Asimismo, esta situación ubica la gestión de conservación y protección integral del ambiente desarrollado por este tipo de UA como poco eficiente y desarticulado con los gobiernos locales y regionales. En cuanto a lo señalado en el párrafo anterior, la eficiencia está comprometida por no cumplir con el marco legal vigente, es decir, de no colocar en práctica los instrumentos de gestión que han sido definidos en la Ley $\mathrm{N}^{0}$ 28611. Otro aspecto que se ve comprometido es la participación ciudadana en la fiscalización ambiental y la presentación de denuncia con el objeto de obtener respuestas oportunas, ya que la capacidad de atención y respuesta a las denuncias por tipo contaminación estará limitada (MINAN, 2016b).

Con respecto a los detalles de la desarticulación de la gestión, esta se da entre los gobiernos locales y el Ministerio del Ambiente, a través del Sistema Nacional de Evaluación y Fiscalización Ambiental, y por el Organismo de Evaluación y Fiscalización Ambiental. Este último ente, es quien aprueba las reglas para la atención de los problemas ambientales presentados. En este sentido, lo que se encontró como denuncias recibidas con mayor frecuencia en estas UA y que requieren ser atendidas por tipos de contaminación, son: Deforestación y Quema de Bosques; Agua y 
Aire. Asimismo, en los 5 departamentos de la región de la selva se evidencia esta situación, observándose mayores dificultades en: Amazonas, Loreto y Madre de Dios.

El análisis realizado sobre los datos de la Encuesta Nacional de Municipalidades 2019, que corresponde a la gestión de conservación y protección del ambiente de los cinco departamentos de la región de la selva, permite concluir en:

- Hay una gran brecha entre el número de unidades ambientales (UA) que existen y las que se requieren por unidad administrativa (distrito, provincia y departamento). Situación que se observa a nivel de país, y particularmente en el caso de los departamentos de Amazonas y Loreto, donde ha ocurrido una reducción sobre el número de UA instaladas.

- A pesar de la heterogeneidad o particularidad que existe en el caso de las municipalidades distritales de cada departamento, se puedo evidenciar que los problemas ambientales reportados en las UA, a través de las denuncias por tipo y elemento de contaminación son las mismas en orden de frecuencia e importancia a las que se reportan a nivel nacional, es decir, que cualquiera estrategia de intervención debe considerar trabajar sobre los tipos de contaminación: Sonora, Agua y Aire; y los elementos contaminantes: 1. Crianza de animales domésticos sin control; 2. Aguas residuales; 3. Deforestación y quema de bosques; 4. Acumulación y quema clandestina de rastrojos; y 5. Emisión de gases de vehículos motorizados.

- En cuanto a la calidad de la gestión de conservación y preservación del ambiente, esta se ve reducida por dos aspectos: 1. No colocar en práctica el marco legal vigente, es decir, la Ley $\mathrm{N}^{\circ}$ 28611: "Ley General del Ambiente", (en su artículo 16: "De los instrumentos"; y articulo 17: "De los tipos de instrumentos"); y 2. La falta de articulación de las acciones de conservación y preservación del ambiente impulsadas por los gobiernos locales, y la fiscalización y aplicación de normas e instrumentos realizada por el Ministerio del Ambiente (MINAN). Esta situación está afectando a todos los departamentos de la región de la selva, y en consecuencia limita principalmente: 1 . La participación ciudadana a través de la realización de las denuncias por tipo de contaminación y la búsqueda de soluciones oportunas de la sociedad civil; y 2. La gestión integral de protección y conservación que se desarrolla desde de los departamentos de Amazonas y Loreto, por ser las áreas geográficas donde existen UA que no cuentan con la aplicación de instrumentos y no se desarrollan acciones ambientales.

\section{REFERENCIAS BIBLIOGRAFICAS}

1. Albert, L. (2004). Contaminación ambiental. Origen, clases, fuentes y efectos. Revista de la facultad de salud pública y nutrición, 228-231.

2. Fernández, D. (2020). El Ambicioso Pacto Verde Europeo. Revista Actualidad Jurídica Ambiental, 101, 1-31.

3. Guzmán, P. (2012). Tratados ambientales internacionales y negocios. Universidad Externado de Colombia.

4. Gonzales, G. F., Zevallos, A., Gonzales-Castañeda, C., Nuñez, D., Gastañaga, C., Cabezas, C., ... \& Steenland, K. (2014). Contaminación ambiental, variabilidad climática y cambio climático: una revisión del impacto en la salud de la población peruana. Revista Peruana de Medicina Experimental y Salud Pública, 31(3), 547-556.

5. Instituto Nacional de Estadística e Informática (2019). Perú: Indicadores de Gestión Municipal. Instituto Nacional de Estadística e Informática

6. Instituto Nacional de Estadística e Informática. (2018). Perú: Indicadores de Gestión Municipal. Instituto Nacional de Estadística e Informática

7. Instituto Nacional de Estadística e Informática. (2017). Capitulo: Protección y Conservación del Ambiente. Instituto Nacional de Estadística e Informática

8. Ley Marco del Sistema Nacional de Gestión Ambiental $\mathrm{N}^{\circ} 28245$ (4 de junio de 2004). https://www.minam. gob.pe/wp-content/uploads/2013/10/leySNGA-28245.pdf

9. Ley General del Ambiente $\mathrm{N}^{\circ} 28611$ (13 de octubre de 2005). https://www.minam.gob.pe/wp-content/ uploads/2017/04/Ley-N\%C2\%B0-28611.pdf

10. Ley $\mathrm{N}^{\circ} 29050$. Ley que modifica el literal k) del artículo 5 de la Ley N ${ }^{\circ} 28245$, Ley Marco del Sistema Nacional de Gestión Ambiental (22 de junio de 2007). https:// www.minam.gob.pe/wp-content/uploads/2017/04/ LEY-N\%C2\%B0-29050.pdf

11. Ministerio del Ambiente. (2016a) Objetivos de Desarrollo Sostenible e Indicadores. Ministerio del Ambiente

12. Ministerio del Ambiente. (2016b). Guía del Sistema Nacional de Gestión Ambiental. Ministerio del Ambiente.

13. Ministerio de la Producción. (2014). Plan Nacional de Diversificación Productiva. Ministerio de la Producción

14. Vargas, L., \& Velásquez, G. (2014). El surgimiento de 
la política global ambiental. Revista Opera, 15 (5), $107-125$

15. Vargas, J. (2008). Economía política ambiental global e internacional. Ra Ximhai: revista cientifica de sociedad, cultura y desarrollo sostenible, 4(1), 83118.

Fecha de recepción: 15 de abril del 2021

Fecha de aceptación: 12 de noviembre del 2021 